\title{
Long-term effect of cardiac resynchronisation in patients reporting mild symptoms of heart failure: a report from the CARE-HF study
}

\author{
J G F Cleland, ${ }^{1}$ N Freemantle, ${ }^{2}$ J-C Daubert, ${ }^{3}$ W D Toff, ${ }^{4}$ F Leisch, ${ }^{5}$ L Tavazzi ${ }^{6}$
}

${ }^{1}$ University of Hull, Kingston upon Hull, UK; ${ }^{2}$ University of Birmingham, Edgbaston, UK; ${ }^{3}$ Hôpital Pontchaillou, Rennes, France; ${ }^{4}$ University Hospitals of Leicester NHS Trust, Leicester, UK; ${ }^{5}$ Academic Hospital Linz, Linz, Austria; ${ }^{6}$ IRCCS Policlinico S. Matteo, Pavia, Italy

Correspondence to:

Professor John G F Cleland, MD, Department of Cardiology, University of Hull, Castle Hill Hospital, Kingston-upon-Hull, UK; g.m.porter@hull.ac.uk

Accepted 9 October 2007 Published Online First 5 Nov 2007

\begin{abstract}
Background: Cardiac resynchronisation therapy (CRT) improves symptoms and prognosis in patients with heart failure and cardiac dyssynchrony. Guidelines from the National Institute of Health and Clinical Excellence in the United Kingdom recommend CRT for patients with recent or persistent moderate or severe symptoms of heart failure. This analysis investigated whether the severity of symptoms was an important determinant of the prognostic benefits of CRT.

Methods: In CARE-HF, patients with left ventricular ejection fraction $\leqslant 35 \%$ and markers of cardiac dyssynchrony who were, in the investigators' opinion, in New York Heart Association (NYHA) class III/IV were randomly assigned to pharmacological treatment alone or with addition of CRT. This analysis investigated whether the severity of symptoms reported by patients, using Likert Scales from the EuroHeart Failure Questionnaire and selfassessed NYHA class, influenced prognosis and the response to CRT.

Results: Of 813 patients, 175 (21.5\%) assessed themselves to be in NYHA class I or II. These patients also reported less severe symptoms and better quality of life than patients who assessed themselves to be in NYHA class III or IV. No statistical interaction was observed between the severity of symptoms assessed in several ways and the benefits of CRT on morbidity and mortality. Conclusions: The severity of symptoms was not an important determinant of the prognostic effects of CRT in patients with moderate or severe LVSD and markers of dyssynchrony in the CARE-HF study. This finding requires confirmation in an adequately powered prospective randomised controlled trial in patients with milder symptoms.
\end{abstract}

A series of well-designed randomised controlled trials shows that cardiac resynchronisation therapy (CRT) improves ventricular function, symptoms, quality of life, exercise capacity and prognosis in patients with heart failure due to left ventricular systolic dysfunction who have evidence of cardiac dyssynchrony. ${ }^{1-4}$ All but two of these trials, ${ }^{56}$ however, specified that only patients with moderate or severe functional limitation (New York Heart Association (NYHA) III/IV) should be enrolled.

The CONTAK study randomised, in a doubleblind fashion, 490 patients with heart failure, left ventricular ejection fraction $\leqslant 35 \%$ and ORS $\geqslant 120 \mathrm{msec}$ who required an implantable cardiac defibrillator (ICD) to a device with CRT turned on or off. ${ }^{5}$ About one-third of the patients were in NYHA class I or II. The study failed to show a reduction in the progression of heart failure over the 6-month follow-up but did show an improvement in exercise capacity. The effects on symptoms and exercise capacity were clearest in patients with NYHA class III/IV heart failure, with a nonsignificant effect in NYHA I/II patients with heart failure. On the other hand, improvement in cardiac function was similar regardless of NYHA class. The MIRACLE-ICD-II study enrolled 186 patients exclusively with NYHA class II heart failure, left ventricular ejection fraction $\leqslant 35 \%$ and $\mathrm{ORS}$ $\geqslant 130 \mathrm{msec}$ who were considered candidates for CRT-D and randomly allocated patients to have the device switched on or off for 6 months. ${ }^{6}$ This study failed to show that CRT improved exercise capacity and suggested little effect on quality of life, but did show improvement in symptoms and left ventricular function with CRT.

Whereas one of the principal objectives of treating severe heart failure is the short-term relief of symptoms, the purpose of treating patients with milder symptoms is mainly to retard or prevent long-term progression or reduce the risk of sudden death. It may be difficult to show that symptoms that are already mild improve with therapy. Accordingly, long-term follow-up is important for the proper clinical evaluation of the benefits of therapy in patients with initially mild symptoms to observe the effects on disease progression. The CARE-HF study asked investigators to enrol patients with NYHA class III/IV heart failure and evidence of cardiac dyssynchrony and follow them up for at least 18 months. ${ }^{7}$ Symptoms and quality of life were assessed in detail at baseline, which permitted investigation of symptom severity from the patients' perspective and its relationship to prognosis and the effects of CRT.

\section{METHODS}

The Cardiac Resynchronization-Heart Failure (CARE-HF) trial was a multi-centre, international, randomised trial comparing the effect of implanting or not implanting a CRT device in patients with heart failure in sinus rhythm with a left ventricular ejection fraction $\leqslant 35 \%$ and markers of cardiac dyssynchrony receiving standard pharmacological therapy, generally including loop diuretics, ACE inhibitors, $\beta$-blockers and, for more severe cases, spironolactone. ${ }^{37}$ The protocol specified that only patients considered by the investigator to be in NYHA class III or IV were to be enrolled. CRT devices without a defibrillator function were used. The patients and investigators were not blinded to treatment allocation, since the 
control group did not receive a device, but the members of the end-points committee were kept unaware of the treatment assigned by censoring of relevant documents. Further details of the design, inclusion criteria, baseline clinical and echocardiographic characteristics and the main results have been published. ${ }^{3} 48$

The steering committee (see Acknowledgements) designed the trial. The Medtronic Corporation funded the trial and provided a study manager to supervise its conduct. The sponsor had no access to the database and did not participate in the analysis. All analyses were performed by one of the authors with the assistance of a statistician independent of the sponsor.

The study was approved by the local ethics committee of each participating institution and by appropriate national ethics committees. All patients provided written informed consent, and gave verbal consent prior to randomisation to ensure that they were still willing to participate.

Baseline clinical data, echocardiograms and bloods for assessment of renal function and N-Terminal-pro-Brain Natriuretic Peptide (NT-pro-BNP) were collected on the day of randomisation. Key information was sent to an independent randomisation centre prior to assigning patients to the CRT or control group. Device implantation was planned to occur within 48 hours of randomisation. Patients were then reviewed prior to discharge, at 1 month, at 3 months and then every 3 months for the first year and every 6 months thereafter. Detailed assessments, as at baseline, were repeated only at 3 months and 18 months.

\section{EVALUATION OF SYMPTOMS}

Investigator-assigned NYHA functional class was recorded at enrolment. At baseline, which occurred up to 10 days later, and at 3 months, patients were shown statements corresponding to the four NYHA classes, part of the EuroHeart Failure Survey Questionnaire (EHFSQ), and asked to choose which one they believed best reflected their condition over the previous month. Breathlessness and fatigue during activities of daily living over the previous month were assessed on a six-point scale and overall health on a seven-point scale using the EHFSQ. Quality of life was assessed at baseline and at 3 months using the EQ5D instrument, which asks about symptoms on the day of assessment, and the Minnesota Living with Heart Failure (MLWHF) questionnaire, which asks about symptoms in the previous month. MLWHFQ was reassessed at 18 months. Patients had no special training on how to complete questionnaires.

The relationship between the patient self-assessed and investigator assigned NYHA class was investigated. Then, we compared the clinical characteristics of patients by self-assessed NYHA category, including measures of cardiac function, symptoms and quality of life. We then investigated the interaction between self-assessed NYHA class and the effects of CRT on outcome. Finally, because this was a post hoc analysis, we investigated whether interactions existed between other measures of symptom severity and randomly assigned therapy. We considered that consistent results, regardless of the method of assessing symptoms and outcome, would add validity to the observations. Accordingly, patients were grouped according to the severity of their symptoms of breathlessness or fatigue as "none", "very little", "a little" or "some" (grades 1-4) versus "a lot" or "very much" (grades 5-6) or who rated their overall health as "very good", "good", "quite good" or "average" (grades 1-4) compared with "quite poor", "poor" or "very poor" (grades 5-7) using EHFSQ. Groups were chosen because they made clinical sense but also divided the population roughly into two equal halves.

\section{OUTCOMES OF INTEREST}

Four outcomes of interest were considered: the primary outcome of the main study, all-cause mortality or unplanned hospitalisation for a major cardiovascular event; the primary outcome of the extended study, all-cause mortality; a secondary outcome of the main study, all-cause mortality or hospitalisation for heart failure; and the proportion of patients at 18 months who were alive and with a MLWHF score $\leqslant 35$ corresponding to scores usually found amongst patients in NYHA class I or II. ${ }^{6}$

\section{STATISTICS}

Continuous data were compared using the Kruskal-Wallis test, and proportions were compared using Fisher's exact test. Time to event data were described using Kaplan-Meier curves, and analysed using Cox constant proportional hazards models. Generalised linear models, and mixed models, were constructed using appropriate link functions and error structures. All analyses were conducted in SAS 9.1 (SAS Institute, Cary, NC).

\section{RESULTS}

The main results of the CARE-HF study and its extension phase have been reported. Briefly, 813 patients were randomly assigned to receive or not to receive a CRT device. Investigators assigned 763 patients to NYHA class III and 50 patients to NYHA class IV at enrolment. At baseline, which occurred up to 10 days after enrolment, only $67 \%$ of patients assigned by investigators to NYHA class III and $64 \%$ assigned to class IV agreed with this assessment. Of patients assigned to NYHA III, 23\% felt that NYHA I or II was a more appropriate description of their functional status (fig 1). Overall, using EuroHeart Failure Scores, 49\% of patients reported that they were troubled by breathlessness and $43 \%$ by fatigue "a lot" or "very much" during activities of daily living, while 53\% ranked their health in the worst three categories.

As expected, compared with patients in NYHA class III/IV, fewer patients with self-reported NYHA class I/II heart failure indicated that their breathlessness or fatigue was an important problem, although even amongst patients classifying themselves as NYHA III/IV only a slight majority reported symptoms that troubled them "a lot" or "very much" (table 1). Self-reported NYHA class I/II patients also recorded better quality of life. Median MLWHF score in this group was similar to or better than that reported from patients in NYHA class II heart failure
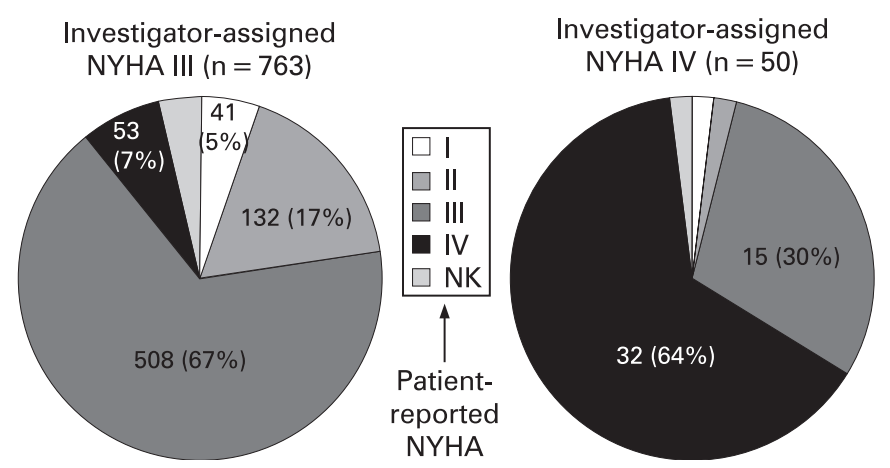

Figure 1 Proportion of patients assigning themselves to different NYHA classes according to investigator-assigned NYHA class at baseline. 
in other studies. ${ }^{6}$ Self-reported NYHA class I/II patients were younger and had higher glomerular filtration rate (GFR) but measures of cardiac dysfunction were similar at baseline to those in patients with more severe symptoms (table 1). The proportion of patients on higher-dose diuretics was similar in patients in NYHA class I/II and III/IV, indicating that more intense diuretic therapy did not account for fewer symptoms. Analysis of patients grouped according to the severity of their breathlessness showed that patients with milder symptoms had higher systolic blood pressure and lower plasma concentrations of NT-pro-BNP despite lower diuretic doses and less severe mitral regurgitation but similar left ventricular ejection fraction to those with more severe symptoms.

In the control group, compared with patients in NYHA class III/IV, the proportion who assigned themselves to NYHA class $\mathrm{I} / \mathrm{II}$ and reached the primary outcome for the main study (49\% versus $58 \% ; p=0.23$ ) or who died during a median follow-up of 37.6 months $(30 \%$ versus $41 \% ; p=0.11)$ was only slightly lower. However, patients who reported themselves to be in NYHA I/II at baseline who survived to 18 months were much more likely to be classified by investigators as being in NYHA class I/II $(60 \%$ vs $31 \%$; $p<0.001)$.

The main outcomes in the overall population are shown in table 2. There was little evidence of an interaction between treatment assignment and patient self-reported NYHA class for any of the selected outcomes (fig 2). The lack of interaction between patient self-reported NYHA class and treatment might reflect the relatively small proportion of patients in NYHA I/II. Alternatively, it might reflect the limitations of patient-reported NYHA class as a measure of symptom severity. Many patients who reported themselves to be in NYHA class I/II nonetheless reported moderate to severe symptoms while many patients who reported themselves to be in NYHA III/IV did not. Accordingly, further analyses according to the severity of symptoms and perceived health and quality of life were conducted (figs 2, 3 and 4). Approximately $50 \%$ of patients were in the worst two categories for symptoms of breathlessness or fatigue and in the worst three categories for perceived health. Further analyses using these measures of symptom severity also failed to identify an interaction with treatment assigned.

\section{DISCUSSION}

This analysis suggests that CRT reduces morbidity and mortality rates in patients with heart failure and cardiac dyssynchrony even when their self-reported symptoms are not severe. This suggests that for many patients CRT, like $\beta$ blockers, may be best targeted at the underlying disease rather than being confined to the treatment of patients with symptoms intractable to pharmacological therapy. Patients with more severe symptoms at baseline may gain more symptom benefit from CRT, at least as judged by the MLWHF questionnaire. This is not unexpected. When improving symptoms is the primary goal of CRT, then their severity and resistance to pharmacological treatment remain important criteria for patient selection. Patients with mild symptoms are unlikely to gain short-term symptomatic benefit, although anecdotally some patients seem unaware of how restricting their symptoms are until they receive treatment. However, when the intention of implanting a CRT device is to modify prognosis, strict application of criteria for symptom severity may not be appropriate.

The CARE-HF protocol, as did COMPANION's, ${ }^{1}$ requested investigators to recruit patients in NYHA functional class III or

Table 1 Baseline characteristics according to patient-assigned NYHA class

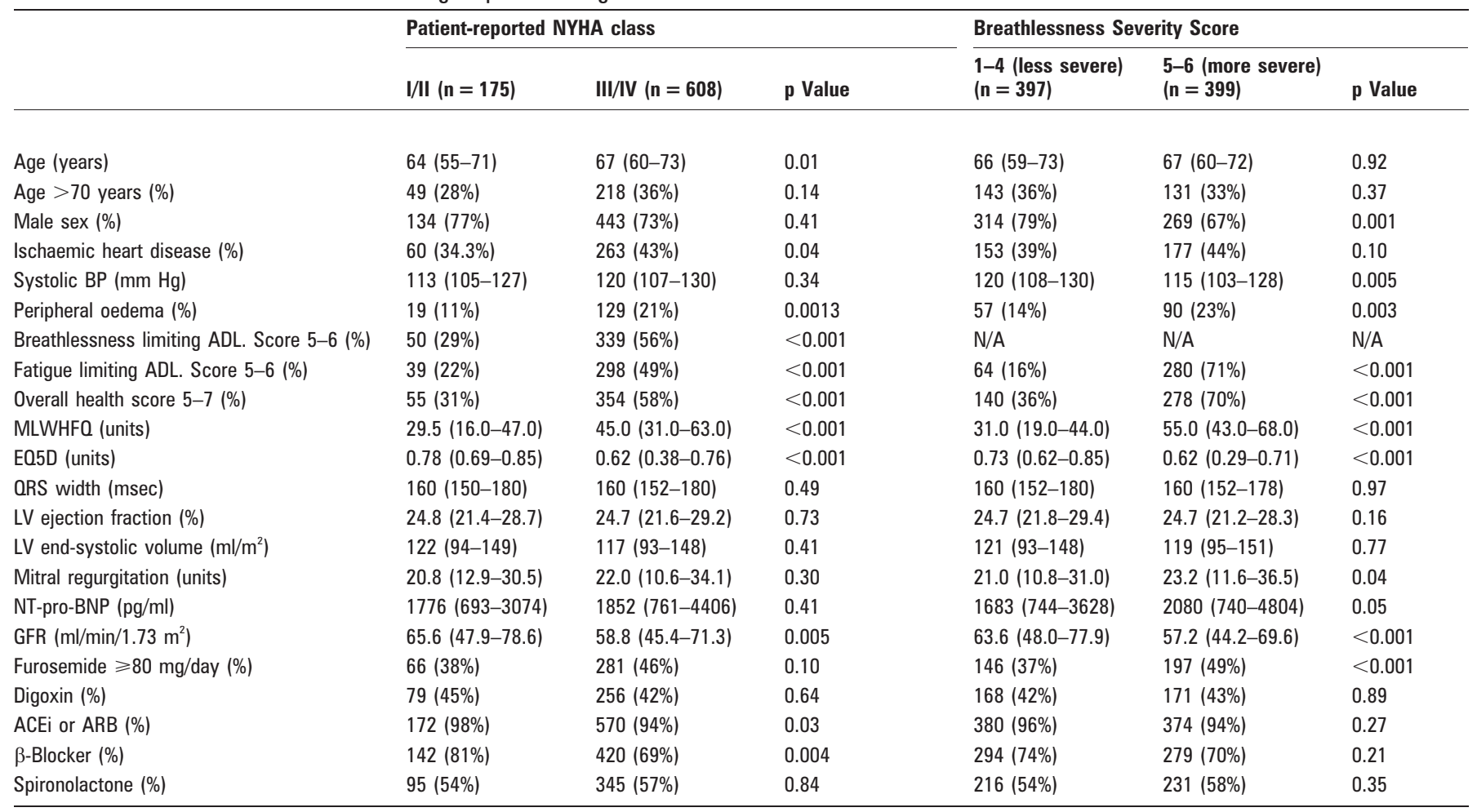

Data shown are percentages or median and inter-quartile range.

$\mathrm{ACEi}$, angiotensin converting enzyme inhibitor; ADL, activities of daily living; $\mathrm{ARB}$, angiotensin receptor blocker; $\mathrm{BP}$, blood pressure; E05D, Euroqol quality of life score; GFR, glomerular filtration rate; LV, left ventricular; MLWHFQ, Minnesota living with heart failure questionnaire; N/A, analysis not appropriate; NT-pro-BNP, N-terminal pro-brain natriuretic peptide. 
A

Overall (159/409 vs 224/404) NYHA I/II (31/86 vs 44/89) NYHA III/IV (124/306 vs 175/302) Breathless < $5(64 / 196$ vs 91/201) Breathless $\geqslant 5(93 / 202$ vs $129 / 197)$ Fatigue $<5(76 / 226$ vs $120 / 224)$ Fatigue $\geqslant 5(81 / 173$ vs $100 / 173)$ Overall Health $<5(64 / 185$ vs $87 / 187)$ Overall Health $\geqslant 5(91 / 212$ vs $132 / 208)$

$\begin{array}{lllll} & & & & \\ 0.2 & 0.5 & 1 & 2\end{array}$

\section{$\widetilde{\text { Favours CRT }}$}

C

Overall (118/409 vs 191/404) NYHA I/II (25/86 vs 39/89) NYHA III/IV (89/306 vs $148 / 302)$ Breathless $<5$ (45/196 vs 79/201) Breathless $\geqslant 5(71 / 202$ vs $108 / 197)$ Fatigue $<5(59 / 226$ vs $103 / 224)$ Fatigue $\geqslant 5(57 / 173$ vs $84 / 173)$ Overall Health $<5(46 / 185$ vs $74 / 187)$ Overall Health $\geqslant 5(68 / 212$ vs $113 / 208)$

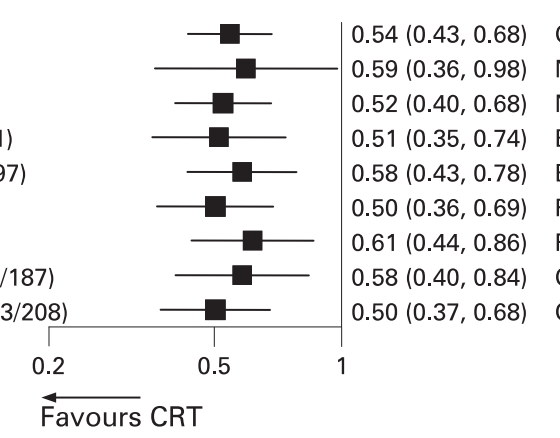

B

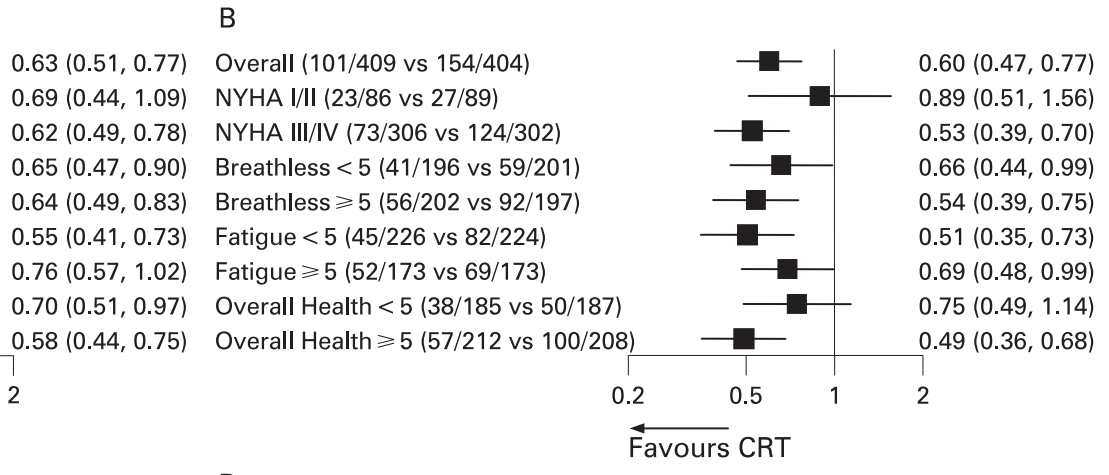

D

Overall (213/409 vs 166/404) NYHA I/II (50/86 vs 52/89) NYHA III/IV (156/306 vs 108/302) Breathless < $5(131 / 196$ vs $117 / 201)$ Breathless $\geqslant 5$ (78/202 vs 49/197) Fatigue $<5(135 / 226$ vs $129 / 224)$ Fatigue $\geqslant 5(75 / 173$ vs $37 / 173)$ Overall Health $<5(118 / 185$ vs $108 / 187)$ Overall Health $\geqslant 5(92 / 212$ vs $55 / 208)$

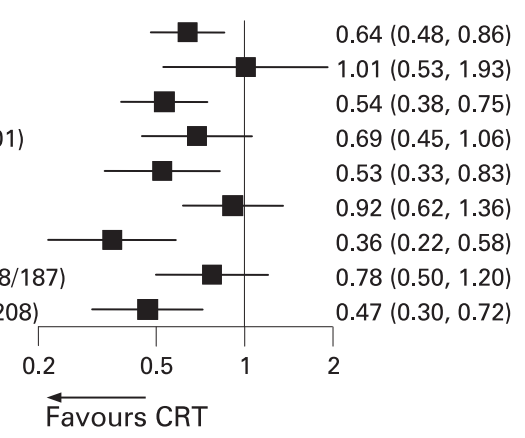

Figure 2 Hazard ratios according to treatment assignment in the overall study and for subgroups according to symptom severity for four outcome measures: (A) the primary outcome of the main study, all-cause mortality or hospitalisation for a major cardiovascular event. No significant interactions observed; (B) the primary outcome of the extended study, all-cause mortality. No significant interactions observed; (C) a secondary outcome of the main study, all-cause mortality or hospitalisation for heart failure. No significant interactions observed; (D) the outcome of being alive with a Minnesota living with heart failure score of $<35$ at 18 months into the study. Only the interaction between fatigue and this outcome was significant $(p=0.0021)$. This reflected a high proportion of patients assigned to either group who had less severe fatigue at baseline reporting MLWHF score $<35$ at 18 months.

IV. However, patients in the control group of CARE-HF had a substantially lower one-year mortality than patients in COMPANION (12.6\% versus 19\%). This could reflect better clinical management of patients in Europe than in the United States, but we consider this an improbable explanation. A more likely explanation was the recruitment of patients with less severe disease in CARE-HF. In patients with fluctuating symptoms, investigators may have been biased to assigning their worst NYHA class to increase enrolment to the study. More than $20 \%$ of the patients in CARE-HF assigned themselves to NYHA class I or II and these patients reported less severe symptoms and impairment of quality of life even though their cardiac function was similar to that of patients who rated themselves worse. Similar discrepancies have been reported in other studies. ${ }^{9}$ Patients in NYHA class I or II were not more likely to be taking high-dose diuretics or other treatment likely to improve symptoms and they had better renal function. These features suggest that more intense therapy did not account for less severe symptoms. More patients were taking $40 \mathrm{mg}$ of furosemide or equivalent per day than any other dose regardless of NYHA grouping in CARE-HF. It is possible that many patients could have been symptomatically improved just by increasing the diuretic dose. Indeed, $40 \%$ of patients in the control group reported that they were in NYHA class I/II by 3 months into the study. ${ }^{3}$

Symptoms are necessarily the patients' subjective experience of disease and will fluctuate over time in response to changes in organ function, activity, mood and expectations. It is naive to consider NYHA class as a fixed or highly reproducible measurement. Investigator-assigned NYHA class is likely to be even more complex than that self-reported by patients since it might be influenced by knowledge of factors such as left ventricular ejection fraction. This may explain why investigator-assigned NYHA class has proved a rather good indicator of prognosis in many studies. Investigators may also have variably classified patients according to their average or worst functional status in the weeks preceding enrolment. Patients were asked to score their average state in the previous month but they may have been disproportionately influenced by their perceived functional status at the time that they completed the questionnaire. There was a gap of a few days between investigator and patient assignment of NYHA class and it is possible that genuine differences in symptom severity emerged during this time. Failure to appreciate the complex issues surrounding symptom assessment may lead to inappropriate application of the entry criteria of clinical trials to clinical practice.

Most current guidelines restrict use of CRT to the minority of patients with heart failure who have persistent moderate or severe symptoms despite modern pharmacological treatment. ${ }^{10}{ }^{11}$ Many patients, however, follow a fluctuating course with periodic exacerbations followed by relative symptom control and stability. Symptoms of heart failure can often be moderately well controlled for most of the time with pharmacological treatment but temporary exacerbations augur a poor prognosis. ${ }^{12}$ The fluctuating course of heart failure can make the timing of intervention difficult. The National Institute of Health and Clinical Excellence in the United Kingdom appears to have recognised the variable nature of symptoms when recommending CRT for patients with moderate or severe symptoms that are either recent or persistent. 


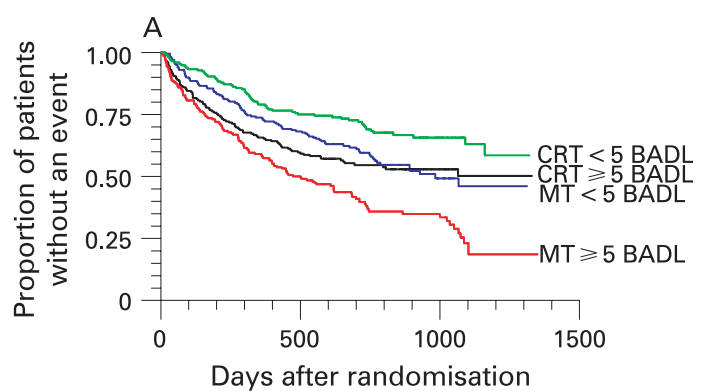

B

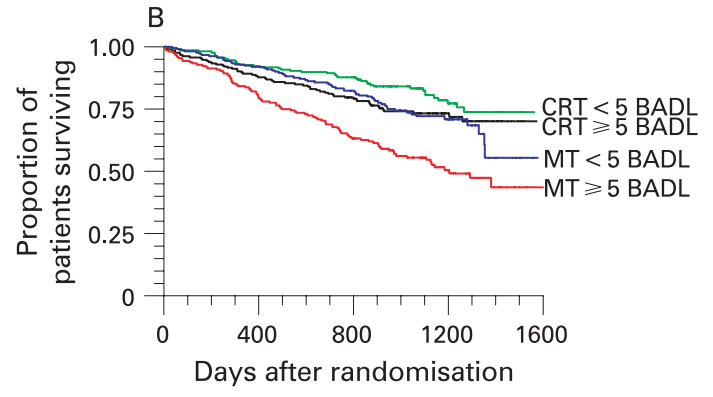

Figure 3 Kaplan-Meier estimates of the time to the primary end point in the main study (death from any cause or unplanned hospitalisation for a major cardiovascular event) (A) and during extended follow-up (allcause mortality) (B) showing the interaction with severity of breathlessness. BADL, breathlessness on activities of daily living $1<5$ indicating milder and $\geqslant 5$ more severe symptoms); CRT, cardiac resynchronisation therapy; MT, pharmacological treatment.

In both the main CARE-HF study and its extension, prespecified subgroup analyses failed to identify a treatment interaction with the severity of cardiac dysfunction as assessed by left ventricular volumes, ejection fraction or NT-pro-BNP. These measurements were objective and evaluated in core laboratories. Few patients reported by investigators to have a LVEF $\leqslant 35 \%$ were found to have values above this threshold in the core laboratory $(n=57,7.6 \%)$. Accordingly, the CARE-HF study cannot provide information on the effect of CRT in patients who do not have severe cardiac dysfunction. Based on the evidence available in this and other trials, it seems likely that CRT is appropriate for patients with a low left ventricular ejection fraction and markers of dyssynchrony if they have experienced moderate or severe symptoms even if their symptoms subsequently improve, provided the intention of treatment is to improve long-term prognosis rather than symptoms.

\section{LIMITATIONS}

This was a retrospectively defined analysis and the findings should be interpreted with caution and treated as hypothesisgenerating. Although we identified groups of patients with relatively mild symptoms, all of the patients had previously been assessed as NYHA class III/IV by an investigator and therefore these data should be extrapolated with caution to the general pool of patients in NYHA class I and II, as determined by a clinician. The effect of CRT on long-term disease progression and LV remodelling in patients with stable mild symptoms, LVEF $\leqslant 40 \%$ and dyssynchrony is currently being investigated in the REVERSE study, although this may not have the duration or event rate required to show conclusive clinical benefit in this population. ${ }^{13}$

In conclusion, the functional assessment of disease severity by clinicians using the NYHA classification may not always
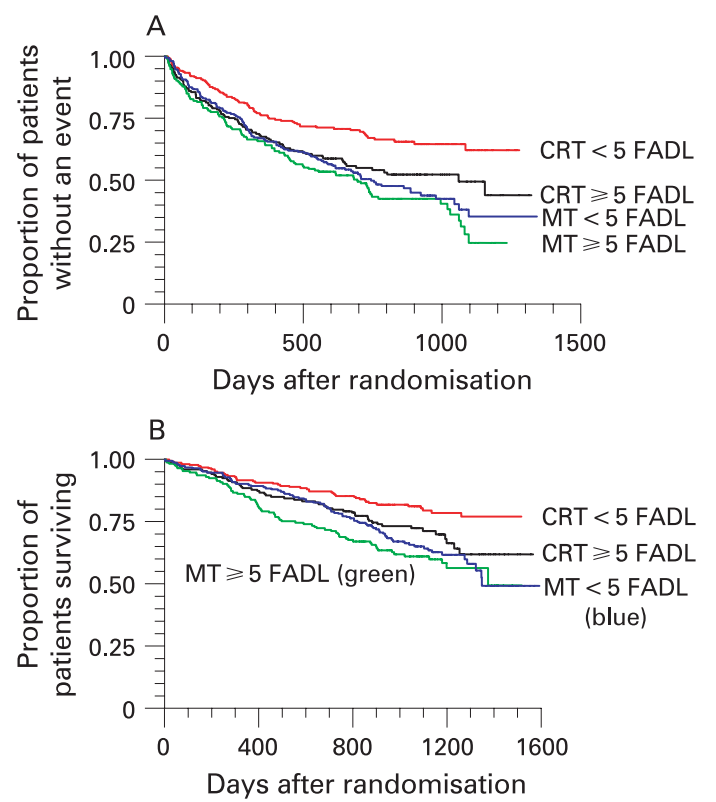

Figure 4 Kaplan-Meier estimates of the time to the primary end point in the main study (death from any cause or unplanned hospitalisation for a major cardiovascular event) (A) and during extended follow-up (allcause mortality) (B) showing the interaction with severity of fatigue. CRT, cardiac resynchronisation therapy; FADL, fatigue on activities of daily living $(<5$ indicating milder and $\geqslant 5$ more severe symptoms); MT, pharmacological treatment.

accord with patients' perceptions of functional class or symptom severity and may fluctuate markedly over short periods of time. Amongst patients recruited to the CARE-HF trial, all of whom were assessed by the investigators to be in NYHA class III or IV, the patient-reported severity of symptoms varied greatly but had little influence on the benefits of CRT on morbidity and mortality. Accordingly, it may not be appropriate to select patients for CRT on the basis of the severity of their symptoms in the presence of persistent marked left ventricular dysfunction and markers of dyssynchrony when the objective of treatment is to improve long-term outcomes. As has already happened with a number of pharmacological treatments for heart failure, such as ACE inhibitors, $\beta$-blockers and aldosterone antagonists, CRT may evolve from a treatment deployed after all others have failed to one that is used early in the course of disease to retard its progression.

Table 2 Outcome by randomised therapy in the overall population

\begin{tabular}{|c|c|c|c|c|}
\hline \multirow[b]{2}{*}{ n } & \multirow{2}{*}{$\frac{\text { Control }}{404(\%)}$} & \multirow{2}{*}{$\frac{\text { CRT }}{409(\%)}$} & \multirow[b]{2}{*}{ HR (95\% CI) } & \multirow[b]{2}{*}{ p Value } \\
\hline & & & & \\
\hline Death or CV hosp (main study) & $224(55)$ & $159(39)$ & $0.63(0.51$ to 0.77$)$ & $<0.001$ \\
\hline Death or HF hosp (main study) & $191(47)$ & $118(29)$ & $0.54(0.43$ to 0.68$)$ & $<0.001$ \\
\hline All-cause mortality* & $154(38)$ & $101(25)$ & $0.60(0.47$ to 0.77$)$ & $<0.001$ \\
\hline Sudden death* & $54(13.4)$ & $32(7.8)$ & $0.54(0.35$ to 0.84$)$ & 0.005 \\
\hline \multicolumn{5}{|l|}{ By 18 months } \\
\hline Investigator-assigned NYHA I/II & $151(37)$ & $255(62)$ & $0.36(0.27$ to 0.48$)$ & $<0.001$ \\
\hline MLWHF Score $\leqslant 35$ & $166(41)$ & $213(52)$ & $0.64(0.48$ to 0.86$)$ & 0.002 \\
\hline CRT implanted & $33(8)$ & $390(95)$ & $\mathrm{N} / \mathrm{A}$ & $\mathrm{N} / \mathrm{A}$ \\
\hline
\end{tabular}

Numbers and percentages are shown for each outcome.

CRT, cardiac resynchronisation therapy; CV hosp, cardiovascular hospitalisation; HF hosp, heart failure hospitalisation; MLWHFO, Minnesota living with heart failure questionnaire. N/A, analysis not appropriate.

*Includes data from both the main study and extension phase. 
Acknowledgements: Steering Committee - JGF Cleland (Chairman), J-C Daubert, E Erdmann, D Gras, L Kappenberger, W Klein, L Tavazzi;

Data and Safety Monitoring Committee - PA Poole-Wilson, L Rydén (Chairman), H Wedel, HJJ Wellens;

Study Management - MMH Marijianowski;

Statistical Analysis Group - N Freemantle, MJ Calvert;

Pharmacovigilance and Data Management - Quintiles;

CARE-HF investigators - Austria - G Christ, F Fruhwald, R Hofmann, A Kypta, F Leisch, R Pacher, F Rauscha; Belgium - R Tavernier; Denmark - PE Bloch Thomsen, S Boesgaard, H Eiskjær, GT Espersen, J Haarbo, A Hagemann, E Korup, M Møller, P Mortensen, P Søgaard, T Vesterlund; Finland - H Huikuri, KI Niemelä, L Toivonen; France - F Bauer, A Cohen-Solal, C Crocq, P Dijiane, JL Dubois-Rande, P de Groote, Y Juilliere, G Kirkorian, M Komajda, T Laperche, H Le Marec, C Leclercq, C Tribouilloy; Germany - F Er, E Fleck, UC Hoppe, FX Kleber, B Maisch, J Neuzner, C Reithmann, T Remp, C Schmitt, C Stahl, RH Strasser; Italy - MC Albanese, A Bartoloni, M Bocchiardo, A Capucci, A Carboni, A Circo, M Disertori, R del Medico, T Forzani, M Frigerio, A Gavazzi, M Landolina, M Lunati, S Mangiameli, M Piacenti, A Pitì, PA Ravazzi, A Raviele, M Santini, A Serio, GP Trevi, M Volterrani, M Zardini; Netherlands FALE Bracke, CC de Cock, A Meijer, R Tukkie; Spain - J Casares Mediavilla, M Concha, JF Delgado, A González-García, R Muñoz-Aguilera, J Martínez Ferrer, F Ridocci; Sweden - B Andren, J Brandt, P Blomström, M Edner, K Hellström, S Jensen, B Kristensson, F Maru, SJ Moller, F Rönn, P Smedgård, G Wikström; Switzerland - J Fuhrer, G Girod; UK - GH Broomes, S Chalil, H Dargie, W Davies, A Delaney, P Elliott, GK Goode, G Haywood, GC Kaye, AS Kurbaan, R Lane, T Levy, F Leyva, H Marshall, S Muhyaldeen, N Nikitin, MJD Roberts, JD Skehan, WD Toff, DJ Wright.

Competing interests: None declared.

\section{REFERENCES}

1. Bristow MR, Saxon LA, Boehmer J, et al, for the comparison of medical therapy, pacing and defibrillation in heart failure (COMPANION) investigators. Cardiacresynchronization therapy with or without an implantable defibrillator in advanced chronic heart failure. New Engl J Med 2004;350:2140-50.

2. Freemantle $\mathbf{N}$, Tharmanathan $\mathrm{P}$, Calvert MJ, et al. Cardiac resynchronisation for patients with heart failure due to left ventricular systolic dysfunction : a systematic review and meta-analysis. Eur J Heart Fail 2006;8:433-40.

3. Cleland JGF, Daubert J-C, Erdmann E, et al, for the Cardiac Resynchronization Heart Failure (CARE-HF) Study Investigatorseart Failure. The effect of cardiac resynchronization on morbidity and mortality in heart failure. New Eng/ J Med 2005;352:1539-49

4. Cleland JGF, Daubert J-C, Erdmann E, et al. Longer-term effects of cardiac resynchronization therapy on mortality in heart failure [the CArdiac REsynchronization - Heart Failure (CARE-HF) trial extension phase]. Eur Heart J 2006;27:1928-32.

5. Higgins SL, Hummel JD, Niazi IK, et al. Cardiac resynchronisation therapy for the treatment of heart failure in patients with intraventricular conduction delay and malignant ventricular tachyarrhythmias. JACC 2003:42:1454-9.

6. Abraham WT, Young JB, Leon AR, et al, on behalf of the multicenter InSync ICD II study group (MIRACLE ICD II). Effects of cardiac resynchronization on disease progression in patients with left ventricular systolic dysfunction, an indication for an implantable cardioverter-defibrillator, and mildly symptomatic chronic heart failure. Circulation 2004;110:2864-8.

7. Cleland JGF, Daubert JC, Erdmann E, et al. On behalf of the CARE-HF Study Steering Committee and Investigators. Design and methodology of the CARE-HF trial. A randomised trial of cardiac resynchronisation in patients with heart failure and ventricular dyssynchrony. Eur J Heart Fail 2001;3:481-9.

8. Cleland JG, Daubert J-C, Erdmann E, et al. On behalf of the CARE-HF study Steering committee and Investigators. Baseline characteristics of patients recruited into the CARE-HF study. Eur J Heart Fail 2005; 7:205-14.

9. Cleland JGF, Goode K, Erhardt L, et al. Carvedilol or Metoprolol European Trial Investigators. A Description of the Clinical Characteristics at Baseline of Patients Recruited into the Carvedilol or Metoprolol European Trial (COMET). Cardiovasc Drugs Ther 2004;18:139-52.

10. Swedberg K, Cleland JGF, Dargie H, et al. Guidelines for the diagnosis and treatment of Chronic Heart Failure. Eur Heart $J$ 2005:26:1115-40.

11. Hunt SA, Abraham WT, Chin MH, et al. ACC/AHA 2005 Guideline Update for the Diagnosis and Management of Chronic Heart Failure in the Adult: A report of the American College of Cardiology/American Heart Association Task Force on Practice Guidelines. Circulation 2005;112:154-235.

12. Cleland JGF, Louis AA, Rigby AS, et al. Noninvasive Home Telemonitoring for Patients With Heart Failure at High Risk of Recurrent Admission and Death. The Trans-European Network-Home-Care Management System (TEN-HMS) Study. J Am Coll Cardiol 2005;45:1654-64.

13. Linde C, Gold M, Abraham WT and Daubert J-C, REVERSE study group. Rationale and design of a randomized controlled trial to assess the safety and efficacy of cardiac resynchronization therapy in patients with asymptomatic left ventricular dysfunction with previous symptoms or mild heart failure - the REsynchronization reVErses Remodeling in Systolic left vEntricular dysfunction (REVERSE) study. Am Heart J 2006:151:288-94. 